\title{
DISCUSSION
}

\section{The effect of calcium ions in pore water on the residual shear strength of kaolinite and sodium montmorillonite}

\author{
R.W.W. ANSON \& A.B. HAWKINS (1998). Géotechnique 48, No. 6, 787-800
}

\begin{abstract}
A. Sridharan, Indian Institute of Science
K. Prakash, Sri Jayachamarajendra College of Engineering The authors are to be complimented for their effort in bringing out the importance of pore medium chemistry on the engineering properties of fine-grained soils, and particularly on residual shear strength. In their introduction, they discuss these effects in detail, as brought out in the literature. However, they do not discuss the role of double layer repulsion, in spite of the fact that it plays an important role in studies of the pore medium chemistry of fine-grained soils, and plenty of information is available in the literature.

Sridharan and co-workers have studied in detail the effect of pore medium chemistry on the volume change behaviour (Sridharan \& Venkatappa Rao, 1973), shear strength (Sridharan \& Venkatappa Rao, 1979) and secondary compression (Sridharan \& Rao, 1982) of fine-grained soils. In their work, to understand the behaviour of soils under a varying physico-chemical environment, they have used the modified effective stress equation
\end{abstract}

$$
\bar{c}=\sigma^{\prime}+\sigma^{\prime \prime}
$$

where $\bar{c}$ is the modified effective stress, $\sigma^{\prime}$ is the conventional effective stress, $\sigma^{\prime \prime}$ is the intrinsic effect stress $=(A-R), R$ is the effective interparticle repulsive pressure, and $A$ is the effective interparticle attractive pressure. Sridharan \& Venkatappa Rao (1979) have shown that the intrinsic effective stress is equal to $c_{\mathrm{d}} / \tan \phi_{\mathrm{d}}$, where $c_{\mathrm{d}}$ is the effective cohesion intercept and $\phi_{\mathrm{d}}$ is the effective angle of shearing resistance obtained in the conventional manner.

Sridharan \& Jayadeva (1982) presented the double layer theory of Gouy-Chapman in a lucid form that could be readily used for understanding the engineering behaviour of clays. Double layer repulsion depends strongly upon dielectric constant, cation valency, cation concentration and, to some extent, the hydrated ionic size. For montmorillonitic soils the interparticle attraction is practically negligible, and their behaviour is controlled primarily by double layer repulsion. For kaolinitic soils double layer repulsion is negligible, and interparticle attraction dominates their behaviour. Thus the engineering behaviour of soils affected by pore medium chemistry can be explained in terms of changes in the electrical interparticle forces brought about by changes in the parameters of pore medium chemistry. Hence, with an increase in the cation concentration and the cation valency, and a decrease in the dielectric constant and hydrated ionic size, double layer repulsion is significantly reduced in montmorillonitic clays. This results in a net increase in the intrinsic effective stress and the modified effective stress, and hence in an increase in the shear strength at the particle level. The results obtained by various researchers, as mentioned by the authors, can be explained consistently in the light of double layer repulsion and the modified effective stress equation.

The dominant decrease in the liquid limit of sodium montmorillonite and, in contrast, the marginal increase in the liquid limit of kaolinite, as observed by the authors, can be explained for sodium montmorillonite in terms of changes in double layer repulsion (Sridharan et al., 1986) and for kaolinite in terms of changes in interparticle attractive forces (Sridharan et al., 1988).

The data represented in Fig. 3 of the paper can be approximated as a straight line for each concentration of $\mathrm{Ca}^{2+}$, as shown in Fig. 11 (only typical plots are given, to save space), and the values of $\phi_{\mathrm{r}}^{\prime}$ and $c_{\mathrm{r}}^{\prime}$ can be obtained. It can be seen from Fig. 12 that the variation in $\phi_{\mathrm{r}}^{\prime}$ over different concentrations of $\mathrm{Ca}^{2+}$ represents only a scatter about a mean value, as there is no definite trend of variation. However, the variation of $c_{\mathrm{r}}^{\prime}$ with the concentration of $\mathrm{Ca}^{2+}$

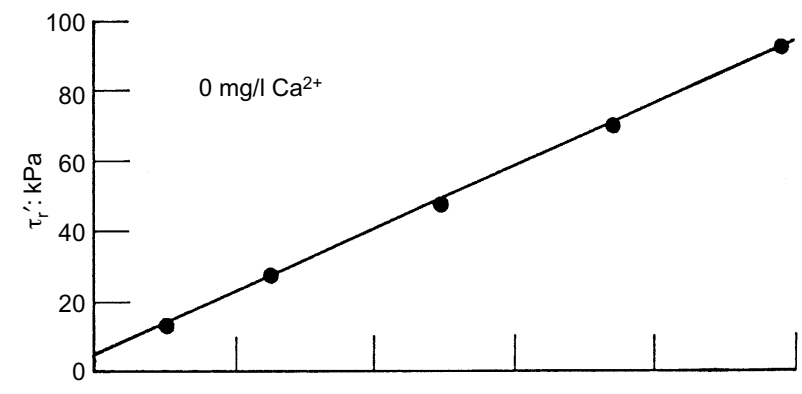

(a)



(b)

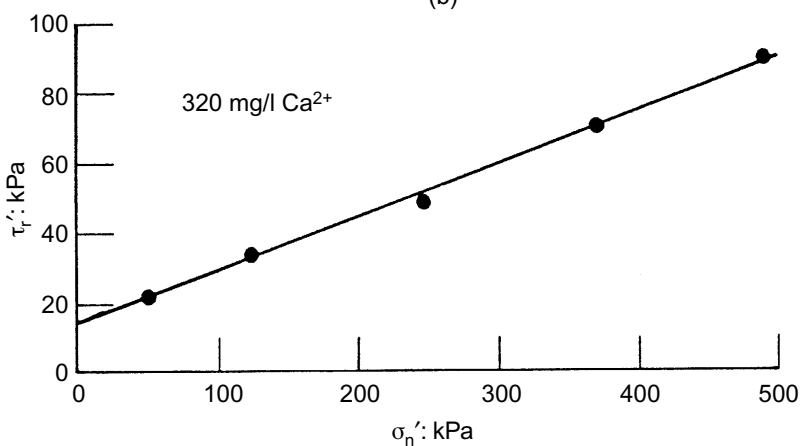

(c)

Fig. 11. Typical residual failure envelopes for kaolinite treated with solutions of different $\mathrm{Ca}^{2+}$ concentrations (for data, see Table 2 of the paper) 


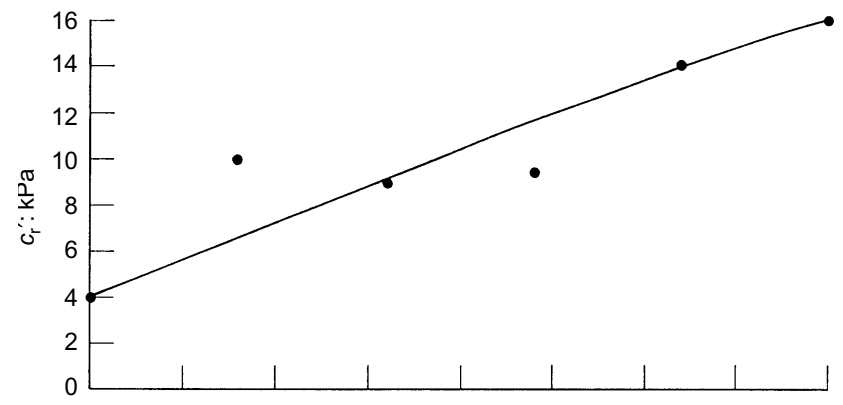

(a)

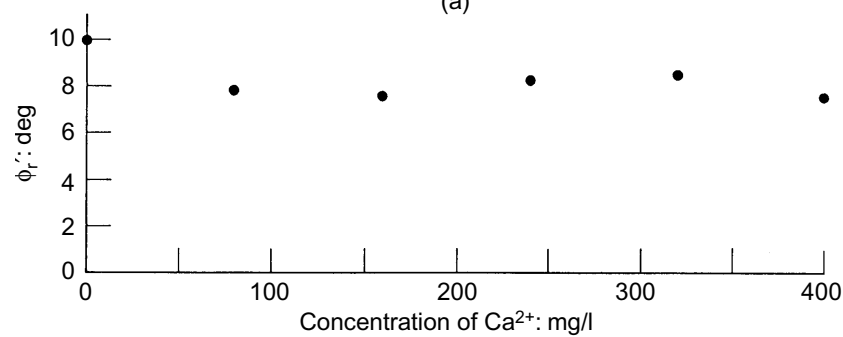

(b)

Fig. 12. Variation of $\phi_{r}^{\prime}$ and $c_{r}^{\prime}$ with concentration of $\mathrm{Ca}^{2+}$ for kaolinite

shows a definite trend in that $c_{\mathrm{r}}^{\prime}$ increases with $\mathrm{Ca}^{2+}$ concentration (Fig. 12). The increase in the cation concentration has resulted in an increase in the attractive pressure (i.e. flocculated fabric), double layer repulsion being negligible in kaolinite. This is responsible for an increase in the modified effective stress, which is manifested through an increase in the values of $c_{\mathrm{r}}^{\prime}$, since the $\sigma_{\mathrm{n}}^{\prime}$ and $\tau_{\mathrm{r}}^{\prime}$ values are plotted in the form of a conventional failure envelope.

In contrast to kaolinite, the results for sodium montmorillonite show altogether different behaviour. Fig. 7 of the paper shows that there is a gradual increase in the values of $\tau_{\mathrm{r}}^{\prime}$ with $\sigma_{\mathrm{n}}^{\prime}$ for each $\mathrm{Ca}^{2+}$ concentration. Such a variation can be approximated as rectangular hyperbolic (typical curves are shown in Fig. 13). This implies that $c_{\mathrm{r}}^{\prime}$ is zero. This can be justified by the fact that $c_{\mathrm{r}}^{\prime}$ is due mainly to interparticle attractive forces in the system, and that the sodium montmorillonite, which is a typical expansive soil, is primarily governed by double layer repulsion, so the attractive forces in the system are negligible.

If one still wants to obtain the residual shear strength parameters at different effective normal stress levels (the writers would prefer to call them $\phi_{\mathrm{t}}^{\prime}$ and $c_{\mathrm{t}}^{\prime}$, not $\phi_{\mathrm{r}}^{\prime}$ and $c_{\mathrm{r}}^{\prime}$ ), then a rectangular hyperbolic approach can be adopted to determine them as indicated below, but only for montmorillonite soils (Fig. 14). In such cases, the following relationship between $\sigma_{\mathrm{n}}^{\prime}$ and $\tau_{\mathrm{r}}^{\prime}$ is valid:

$$
\tau_{\mathrm{t}}^{\prime}=\frac{\sigma_{\mathrm{n}}^{\prime}}{a+b \sigma_{\mathrm{n}}^{\prime}}
$$

where $a$ and $b$ are respectively the intercept on the $\left(\sigma_{\mathrm{n}}^{\prime} / \tau_{\mathrm{r}}^{\prime}\right)$ axis and the slope of the linear portion of the curve of $\left(\sigma_{\mathrm{n}}^{\prime} / \tau_{\mathrm{r}}^{\prime}\right)$ against $\sigma_{\mathrm{n}}^{\prime}$ (Fig. 13). The values of $a$ and $b$ can be used to calculate the values of $\phi_{\mathrm{t}}^{\prime}$ and $c_{\mathrm{t}}^{\prime}$ using the following equations:

$$
\tan \phi_{\mathrm{t}}^{\prime}=\frac{a}{\left(a+b \sigma_{\mathrm{n}}^{\prime}\right)^{2}}
$$

and

$$
c_{\mathrm{t}}^{\prime}=\frac{b\left(\sigma_{\mathrm{n}}^{\prime}\right)^{2}}{\left(a+b \sigma_{\mathrm{n}}^{\prime}\right)^{2}}
$$

Table 8 lists the values of $\phi_{\mathrm{t}}^{\prime}$ and $c_{\mathrm{t}}^{\prime}$ so obtained for various
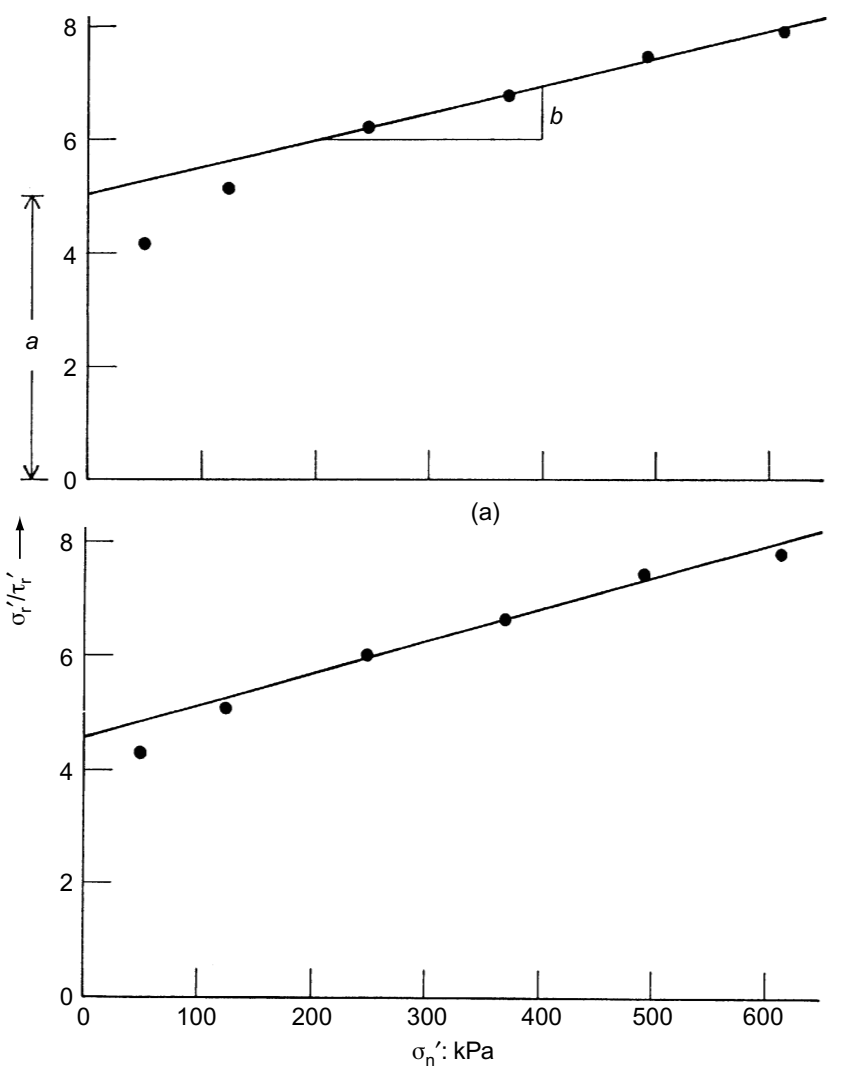

(b)

Fig. 13. Typical curves of $\left(\sigma_{n}^{\prime} / \tau_{n}^{\prime}\right)$ against $\sigma_{n}^{\prime}$ for sodium montmorillonite treated with solutions of different $\mathrm{Ca}^{2+}$ concentrations (for data, see Table 5 of the paper): (a) $160 \mathrm{mg} / \mathrm{l} \mathrm{Ca}^{2+}$; (b) $320 \mathrm{mg} / \mathrm{l} \mathrm{Ca}{ }^{2+}$

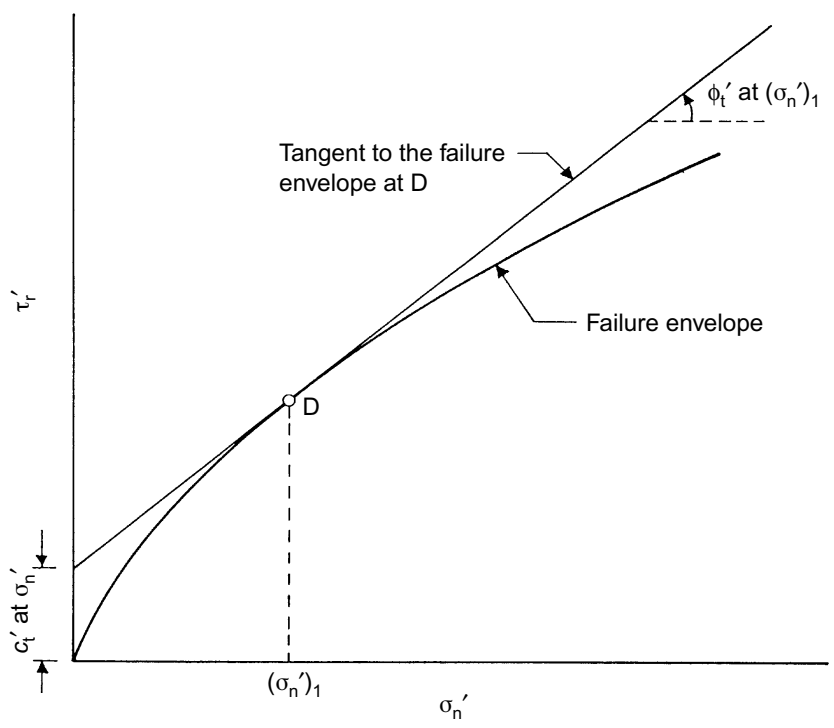

Fig. 14. Schematic diagram indicating the meaning of $\phi_{\mathrm{t}}^{\prime}$ and $c_{\mathrm{t}}^{\prime}$

values of $\sigma_{\mathrm{n}}^{\prime}$ for different concentrations of $\mathrm{Ca}^{2+}$. The writers feel that the rectangular hyperbolic approach for determining the residual shear strength parameters at any effective normal stress level for montmorillonitic soils is more viable than the method adopted by the authors.

Even though it is advisable to use the effective residual cohesion intercept in analysing practical problems, the judgement should be based on the type of clay mineral in the 
Table 8. Values of $\phi_{\mathrm{t}}^{\prime}$ and $c_{\mathrm{t}}^{\prime}$ for sodium montmorillonite at different effective normal stress levels, obtained by the proposed rectangular hyperbolic approach

\begin{tabular}{|c|c|c|c|c|c|c|c|c|c|c|c|c|}
\hline \multirow{2}{*}{$\begin{array}{c}\boldsymbol{\sigma}_{\mathrm{n}}^{1} \\
\mathrm{kPa}\end{array}$} & \multicolumn{2}{|c|}{$0 \mathrm{mg} / 1 \mathrm{Ca}^{2+}$} & \multicolumn{2}{|c|}{$80 \mathrm{mg} / 1 \mathrm{Ca}^{2+}$} & \multicolumn{2}{|c|}{$160 \mathrm{mg} / 1 \mathrm{Ca}^{2+}$} & \multicolumn{2}{|c|}{$240 \mathrm{mg} / 1 \mathrm{Ca}^{2+}$} & \multicolumn{2}{|c|}{$320 \mathrm{mg} / 1 \mathrm{Ca}^{2+}$} & \multicolumn{2}{|c|}{$400 \mathrm{mg} / 1 \mathrm{Ca}^{2+}$} \\
\hline & $\phi_{\mathrm{t}}^{\prime}: \operatorname{deg}$ & $c_{\mathrm{t}}^{\prime}: \mathrm{kPa}$ & $\phi_{\mathrm{t}}^{\prime}: \operatorname{deg}$ & $c_{\mathrm{t}}^{\prime}: \mathrm{kPa}$ & $\phi_{\mathrm{t}}^{\prime}: \operatorname{deg}$ & $c_{\mathrm{t}}^{\prime}: \mathrm{kPa}$ & $\phi_{\mathrm{t}}^{\prime}: \operatorname{deg}$ & $c_{\mathrm{t}}^{\prime}: \mathrm{kPa}$ & $\phi_{\mathrm{t}}^{\prime}: \operatorname{deg}$ & $c_{\mathrm{t}}^{\prime}: \mathrm{kPa}$ & $\phi_{\mathrm{t}}^{\prime}: \operatorname{deg}$ & $c_{\mathrm{t}}^{\prime}: \mathrm{kPa}$ \\
\hline 0 & $4 \cdot 8$ & 0 & $11 \cdot 4$ & 0 & $11 \cdot 3$ & 0 & $10 \cdot 6$ & 0 & $12 \cdot 3$ & 0 & $11 \cdot 4$ & 0 \\
\hline 25 & $4 \cdot 7$ & 0.02 & $10 \cdot 8$ & $0 \cdot 14$ & $10 \cdot 8$ & $0 \cdot 11$ & $10 \cdot 2$ & 0.08 & $11 \cdot 6$ & $0 \cdot 16$ & $10 \cdot 9$ & $0 \cdot 10$ \\
\hline 50 & $4 \cdot 6$ & 0.06 & $10 \cdot 2$ & 0.54 & $10 \cdot 3$ & 0.43 & $9 \cdot 9$ & $0 \cdot 31$ & $10 \cdot 9$ & 0.58 & $10 \cdot 5$ & $0 \cdot 39$ \\
\hline 100 & $4 \cdot 5$ & $0 \cdot 25$ & $9 \cdot 1$ & 1.94 & $9 \cdot 5$ & 1.59 & $9 \cdot 3$ & $1 \cdot 14$ & $9 \cdot 8$ & $2 \cdot 09$ & $9 \cdot 7$ & $1 \cdot 44$ \\
\hline 150 & $4 \cdot 4$ & 0.54 & $8 \cdot 2$ & $3 \cdot 93$ & $8 \cdot 7$ & $3 \cdot 28$ & $8 \cdot 7$ & $2 \cdot 41$ & 8.9 & $4 \cdot 23$ & $9 \cdot 0$ & $3 \cdot 01$ \\
\hline 200 & $4 \cdot 2$ & 0.93 & $7 \cdot 5$ & $6 \cdot 33$ & 8.0 & $5 \cdot 38$ & $8 \cdot 2$ & $4 \cdot 03$ & $8 \cdot 0$ & $6 \cdot 81$ & $8 \cdot 4$ & $4 \cdot 97$ \\
\hline
\end{tabular}

soil encountered. The use of the effective residual cohesion intercept may be recommended when dealing with kaolinitic soils (i.e. non-expanding lattice type of soils), in which the fabric of the soil governed by the interparticle attractive forces plays a dominant role. However, the use of the effective residual cohesion intercept when dealing with montmorillonitic soils (i.e. expanding lattice type of soils) may not be justifiable, as the strength of such soils is controlled by double layer repulsion.

\section{Authors' reply}

The authors acknowledge an interesting contribution to their paper. It is indeed encouraging that other workers take the time to re-evaluate published data and offer a different emphasis. Such reassessment adds value and shows the necessity for workers to provide within their papers sufficient raw data to allow this.

The discussors state that they have drawn attention to additional points that perhaps were not fully developed by the authors. We note, for instance, in the written text that we could have said more on the rise in the cohesion value of kaolinite with increasing calcium concentration, although the tabulated data indicate this trend. It is perhaps not as clear as the discussors imply.

It is regretted that the discussors did not include all their data in Fig. 11, thus preventing the reader from assessing their conclusion from the full range of calcium contents. We accept that in showing all data points it is likely that some, on any graph, will not fit and are not necessarily easy to explain.

The authors are concerned that Table 1 provided by the discussors gives a set of derived parameters $\left(c_{\mathrm{t}}^{\prime}\right)$ that imply a precision inconsistent with real soils and real laboratory testing.

We agree with the discussors that careful consideration is required in the analysis of practical problems and that, as reminded by them, $c_{\mathrm{r}}^{\prime}=0$ may be too conservative except for very shallow failures.

\section{REFERENCES}

Sridharan, A. \& Jayadeva, M. S. (1982). Double layer theory and compressibility of clays, Géotechnique 32, No. 2, 133-144.

Sridharan, A. \& Rao, A. S. (1982). Mechanisms controlling the secondary compression of clays. Géotechnique 32, No. 3, 249260.

Sridharan, A. \& Venkatappa Rao, G. (1973). Mechanisms controlling volume change behaviour of saturated clays and the role of the effective stress concept. Géotechnique 23, No. 3, 359-382.

Sridharan, A. \& Venkatappa Rao, G. (1979). Shear strength behaviour of saturated clays and the role of the effective stress concept. Géotechnique 29, No. 2, 177-193.

Sridharan, A., Rao, S. M. \& Murthy, N. S. (1986). Liquid limit of montmorillonite soils. Geotech. Test. J. 9, 156-159.

Sridharan, A., Rao, S. M. \& Murthy, N. S. (1988). Liquid limit of kaolinitic soils. Géotechnique 38, No. 2, 191-198. 